\title{
MINIMAL TWIN SURFACES
}

\author{
HAO CHEN
}

\begin{abstract}
We report some minimal surfaces that can be seen as copies of a triply periodic minimal surface (TPMS) related by reflections in parallel mirrors. We call them minimal twin surfaces for the resemblance with twin crystal. Brakke's Surface Evolver is employed to construct twinnings of various classical TPMS, including Schwarz' Primitive (P) and Diamond (D) surfaces, their rhombohedral deformations (rPD), and Schoen's Gyroid (G) surface. Our numerical results provide strong evidences for the mathematical existence of $D$ twins and $G$ twins, which are recently observed in experiment by material scientists. For rPD twins, we develop a good understanding, by noticing examples previously constructed by Traizet (2008) and Fujimori and Weber (2009). Our knowledge on G twins is, by contrast, very limited. Nevertheless, our experiments lead to new cubic polyhedral models for the D and G surfaces, inspired by which we speculate new TPMS deformations in the framework of Traizet.
\end{abstract}

\section{INTRODUCTION}

In material science, a crystal twinning refers to a symmetric coexistence of two or more crystals related by Euclidean motions. The simplest situation, namely the reflection twin, consists of two crystals related by a mirror reflection in the boundary plane.

Triply periodic minimal surfaces (TPMS) are minimal surfaces with the symmetries of crystals. They are used to model lyotropic liquid crystals and many other structures in nature. Recently, [Han et al., 2011] synthesized mesoporous crystal spheres with polyhedral hollows; see also [Lin et al., 2017]. A crystallographic investigation reveals the structure of Schwarz' D (diamond) surface. Most interestingly, twinning phenomena are observed at the boundaries of the domains; see Figure 1. We also notice Figure 7.1(b) in [Hyde et al., 1996], which seems like another evidence, but did not catch the attention at the time. Han et al. also observed twinnings of Schoen's G (gyroid) structure $^{1}$, which were earlier discovered by [Vignolini et al., 2012].

However, it is mathematically premature to call the observed structures "minimal twin surfaces". Despite the common belief and various convincing physics explanations, the energetic base of mesophased systems forming periodic minimal surfaces is not well understood. We could not say for sure that the observed surfaces are minimal. Moreover, it is a priori not known, mathematically, that a minimal surface with the observed twin structure exists.

In this note, we report some minimal surfaces that deserve the name "minimal twin surfaces". They are similar to polysynthetic twin crystals, treating TPMS as crystals. More specifically, they look like copies of a TPMS related by mirror reflections in parallel planes. The minimum lattice distance $\delta$ between the reflection planes is a parameter of the twin surface. We will construct such twinnings for rPD surfaces and Schoen's G surface. rPD surfaces are rhombohedral deformations of Schwarz' P and D surfaces; they are parametrized by a positive real number $t>0$.

We use Brakke's Surface Evolver [Brakke, 1992], an efficient gradient descender, for construction. A minimal surface is obtained if we manage to reduce the integral of squared mean curvature down to practically 0 . Our main observation is the following:

Observation 1. rPD twins and $\mathrm{G}$ twins exist for a large set of parameters.

2010 Mathematics Subject Classification. Primary 53A10, 49Q05.

Key words and phrases. Triply periodic minimal surfaces, Twin lattice, Surface Evolver.

A revision of the manuscript was done while the author was in residence at the Mathematical Sciences Research Institute in Berkeley, California, during the Fall 2017 semester, supported by the National Science Foundation under Grant No. DMS-1440140.

${ }^{1}$ Paper in preparation. 


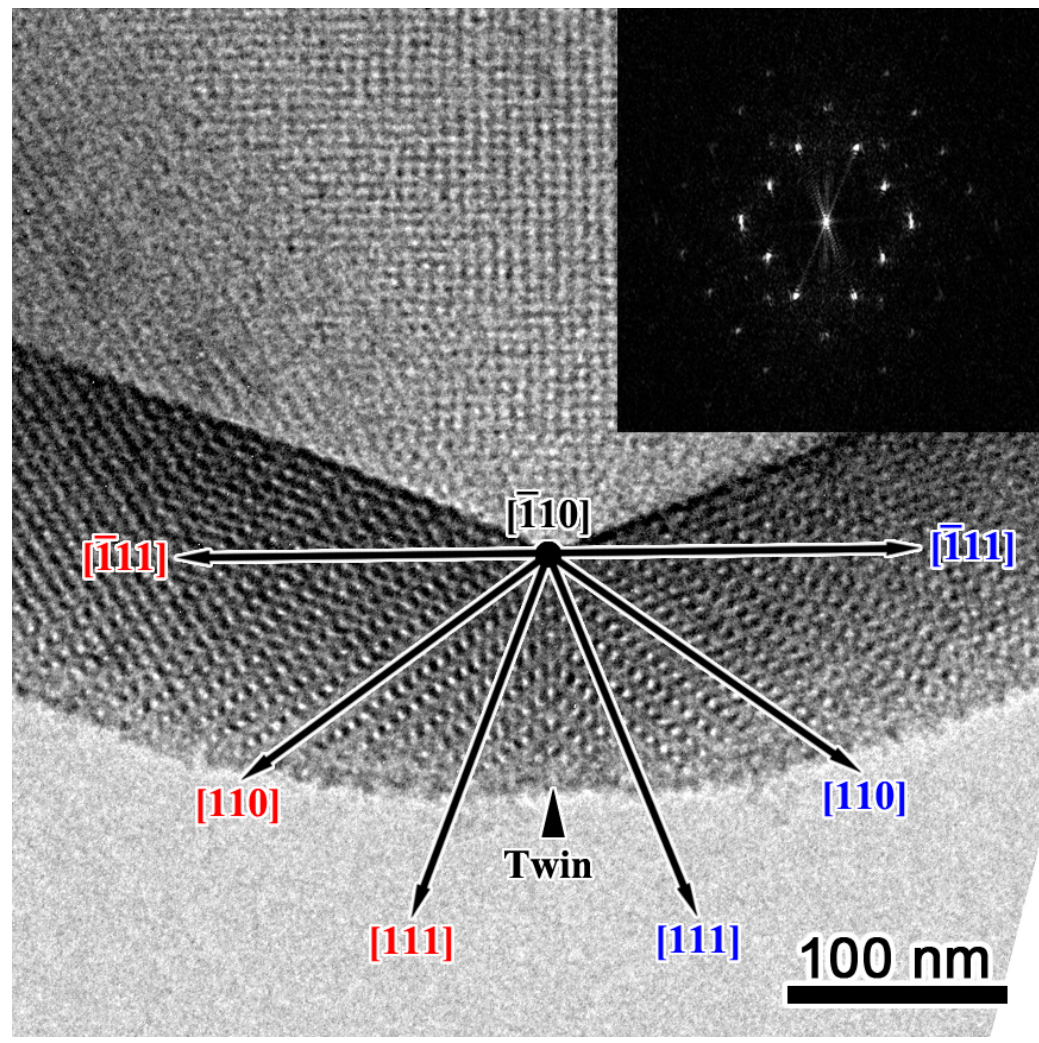

FiguRE 1. Twin structure in minimal D surface observed in experiment. Reuse with permission from [Han et al., 2011]. Copyright (c)2011 American Chemical Society.

In particular, $\mathrm{D}$ twins and $\mathrm{G}$ twins exist for very large $\delta$, providing a strong evidence for the mathematical existence of the twin structures experimentally observed by [Han et al., 2011]. However, our experiment for $\mathrm{rPD}$ twins with large parameter $t$ is not conclusive even for small $\delta$.

For the rPD twins, we notice that extreme examples have been constructed in previous works.

Examples of small $\delta$ was described by [Fujimori and Weber, 2009]. However, the period problem was only solved for $\delta=1$ and $\delta=2$. Following their work, we observe that, when $\delta=3$, an $\operatorname{rPD}$ twin with sufficiently large $t$ (near helicoid limit) does not exist.

On the other hand, examples with sufficiently small $t$ (near catenoid limit) follows from [Traizet, 2008]. His approach reveals an analogy of rPD and H surfaces with the cubic and hexagonal closed packings. Further development of Traizet's technique might lead to rigorous constructions of twin TPMS with a single reflection plane.

Our understanding of the $\mathrm{G}$ twins is very limited. However, our numerical result inspires new cubic polyhedral models for the $\mathrm{G}$ and $\mathrm{D}$ surfaces. This leads to new interpretations of the $\mathrm{D}$ and G surfaces in the framework of [Traizet, 2008]. Based on numerical evidences, we speculate new deformations of the $\mathrm{D}$ and $\mathrm{G}$ surfaces. In particular, we notice a new tetragonal deformation family with the symmetry of the tD family.

This note is organized as follows. In Section 2 we define TPMS twinning in analogy with crystal twinning. In Section 3, we introduce the rPD surfaces, define their twins by Weierstrass representation, and present rigorous examples with small $\delta$ (Section 3.3) and with small $t$ (Section 3.4), as well as numerical examples in Surface Evolver (Section 3.5). The G twins are numerically constructed in Section 4.1. Then we propose new cubic polyhedral models (Section 4.2) and new TPMS deformations (Section 4.3) for the D and G surfaces in the framework of [Traizet, 2008]. 


\section{ACKNOWLEDGEMENT}

This manuscript, while self-contained, presents mathematical context and technical details as a complement and extension to another paper in preparation on material science. I would like to thank my collaborators in that project, especially Chenyu Jin, Lu Han and Nobuhisa Fujita, for their helps on this note. I'm very grateful to Karsten Große-Brauckmann for valuable suggestions, to Matthias Weber for his comments and Mathematica programs, and to Han Yu and Martin Traizet for helpful discussions. Most of the work was done while the author is visiting St Andrews University, and the author thanks Louis Theran for his kind invitation.

\section{CRystal twinning And TPMS twinning}

2.1. Crystals and TPMS. In physics, a crystal is modeled by a Bravais lattice, which can be defined as a discrete point set $\Lambda \subset \mathbb{R}^{3}$ invariant under three linearly independent translations. A lattice plane of $\Lambda$ is a plane $H$ such that $H \cap \Lambda$ is a non-empty 2-dimensional lattice, i.e. invariant under two linearly independent translations.

In this note, the lattice planes of interest are usually placed parallel or orthogonal to the $z$-axis. But from time to time, we will use Miller indices to denote the directions of lattice planes and vectors. Here is a quick reference for readers who are not familiar with this notation.

Let $h, k$ and $l$ be three coprime integers. For a lattice spanned by vectors $\mathbf{a}, \mathbf{b}, \mathbf{c}$

- $[h k l]$ denotes the direction of the vector $h \mathbf{a}+k \mathbf{b}+l \mathbf{c}$;

- $(h k l)$ denotes the lattice planes passing through $n \mathbf{a} / h, n \mathbf{b} / k$ and $n \mathbf{c} / l$, for some integer $n$;

- $\langle h k l\rangle$ denotes directions that are equivalent to $[h k l]$ by symmetry; and

- $\{h k l\}$ denotes lattice planes that are equivalent to $(h k l)$ by symmetry.

Negative numbers in Miller index will be replaced by a number with bar, e.g. $\overline{1}$ for -1 .

Alternatively, an $(h k l)$ lattice plane is the set

$$
H_{n}=\left\{\alpha \mathbf{a}+\beta \mathbf{b}+\gamma \mathbf{c} \in \Lambda \mid(\alpha, \beta, \gamma) \in \mathbb{Z}^{3}, \alpha h+\beta k+\gamma l=n\right\}
$$

for some $n \in \mathbb{Z}$. The lattice distance between two $(h k l)$ lattice planes $H_{n}$ and $H_{m}$ is defined as $|m-n|$. For $H=H_{n}$, we use $H^{+}$(resp. $H^{-}$) to denote the half-space bounded by $H$ containing the parallel lattice planes $H_{m}$ with $m>n$ (resp. $m<n$ ). The normal vector of $H$ is considered as pointing towards $H^{+}$.

A lattice $\Lambda$ can also be seen as a discrete group acting on $\mathbb{R}^{3}$ by translations; the Bravais lattice is just an orbit of this group. A triply periodic minimal surface (TPMS) $S \subset \mathbb{R}^{3}$ is an oriented minimal surface invariant under the action of a lattice $\Lambda$. The quotient $S / \Lambda$ is called the translational unit ${ }^{2}$. A plane $H$ is a lattice plane of $S$ if $H \cap S$ is invariant under the action of a 2-dimensional lattice. Parallel lattice planes have the same translational symmetries.

There are several ways to extract a Bravais lattice $\Lambda$ from a TPMS $S$. For the TPMS that we are interested in (namely rPD and G surfaces), we find it convenient to use flat points as lattice points. Note that a lattice plane $H$ for $S$ may not be a lattice plane for $\Lambda$, because $H$ may contain no flat point at all. Let $\mathbf{a}, \mathbf{b}$ and $\mathbf{c}$ be the generators of $\Lambda$, then a TPMS lattice plane $H$ can be written as the set

$$
H=\left\{\alpha \mathbf{a}+\beta \mathbf{b}+\gamma \mathbf{c} \mid(\alpha, \beta, \gamma) \in \mathbb{R}^{3}, \alpha h+\beta k+\gamma l=r\right\}
$$

for a unique $r \in \mathbb{R}$. Note that the coefficients $\alpha, \beta$ and $\gamma$ are in $\mathbb{R}$ instead of $\mathbb{Z}$. We call the fractional part $\{r\}=r-\lfloor r\rfloor$ the offset of $H . H$ contains flat points if and only if its offset is 0 . The distance between TPMS lattice planes, as well as the half-spaces $H^{ \pm}$, are defined in the same way as for a Bravais lattice.

\footnotetext{
${ }^{2}$ The crystallographic term is "primitive unit cell".
} 
2.2. Twin TPMS and their symmetries. A lattice plane $H$ of a Bravais lattice $\Lambda$ (resp. a TPMS $S$ ) is said to be trivial if $\Lambda$ (resp. $S$ ) is symmetric under the reflection in $H$.

The reflection twin of a Bravais lattice $\Lambda$ about a non-trivial lattice plane $H$ consists of $\Lambda \cap H^{+}$ and its reflective image about $H$ in the half-space $H^{-}$. We call $H$ the twin boundary. Note that this model is idealized. In reality, twinning a lattice in this way will introduce inhomogeneity to the energy, so atoms near the twin boundary will be slightly pulled away from their original positions.

For a non-trivial $H$ of a TPMS $S$, if we take $S \cap H^{+}$and its reflective image in $\mathrm{H}^{-}$, the surface obtained is not smooth on the twin boundary, hence definitely not a minimal surface.

Instead of naively imitating the construction for crystal twinning, we will define TPMS twinning by specifying the symmetry. Like Bravais lattices, TPMS often admit extra symmetries other than the translations. The symmetry group or space group of a TPMS $S$, denoted by $\operatorname{Sym}(S)$, is the group of Euclidean symmetries of $S$. For a lattice plane $H$ of $S, \operatorname{Sym}(H)$ is the (setwise) stabilizer of $H \cap S$ in $\operatorname{Sym}(S)$. In other words, $\operatorname{Sym}(H)$ is the subgroup of $\operatorname{Sym}(S)$ leaving $H \cap S$ (setwise) invariant. For example, the following elements of $\operatorname{Sym}(S)$ belongs to $\operatorname{Sym}(H)$ :

- the translations in directions parallel to $H$,

- the rotations about axis orthogonal to $H$, and

- the reflections in a plane orthogonal to $H$.

Parallel lattice planes have the same symmetry. Crystal twinning breaks symmetries of the lattice, but preserves those symmetries that leave the twin boundary invariant. We expect the same for the TPMS twinning.

We say that a surface $S^{\prime}$ is asymptotic to $S$ in a half-space $H^{+}$if there is a sequence of translations $\left(T_{k}\right)_{k \in \mathbb{N}}$ in the normal direction of $H$ such that $\left(S^{\prime} \cap T_{k}\left(H^{+}\right)\right)_{k \in \mathbb{N}}$ converges to $T_{k}\left(S \cap H^{+}\right)$.

We are now ready to define the TPMS twinning:

Definition 1. Let $S \subset \mathbb{R}^{3}$ be a triply periodic minimal surface, and $H$ be a non-trivial lattice plane of $S$. A reflection twin of $S$ about $H$ is a doubly periodic minimal surface $\Sigma$ that is

- invariant under the action of $\operatorname{Sym}(H)$;

- invariant under the reflection in $H$;

- asymptotic to $S$ in $H^{+}$.

Remark. The anonymous referee points out that the reflection twins are examples of the following general problem: Given two minimal surfaces $S^{+}$and $S^{-}$and a hyperplane $H \subset \mathbb{R}^{3}$, find a minimal surface $\Sigma$ that is asymptotic to $S^{+}$in $H^{+}$and asymptotic to $S^{-}$in $H^{-}$. Earlier examples include the Scherk surface, for which $S^{+}$is parallel horizontal planes, and $S^{-}$is parallel vertical planes.

A reflection twin has a single symmetry plane $H$, hence not periodic in the direction orthogonal to $H$. Available tools for minimal surfaces of infinite topology are very limited. The author is aware of [Morabito and Traizet, 2012] and [Traizet, 2013] which, if combined with the arguments in [Traizet, 2008], could lead to a rigorous treatment of reflection twin. This is the topic of a future project.

The current manuscript focuses on numerical experiments. Then the aperiodicity becomes very inconvenient as computers do not really understand infinity. Therefore, we will work with polysynthetic twins with parallel reflection planes, as an approximation of single reflection twins.

Definition 2. Let $S \subset \mathbb{R}^{3}$ be a triply periodic minimal surface, and $H$ be a non-trivial lattice plane of $S$. A polysynthetic twin of $S$ (or an $S$ twin) about $H$ is a triply periodic minimal surface $\Sigma_{\delta}$, for some integer parameter $\delta>0$, such that

- $\Sigma_{\delta}$ is invariant under the action of $\operatorname{Sym}(H)$;

- $\Sigma_{\delta}$ is invariant under the reflection in $H$ and parallel planes;

- the minimum lattice distance between reflection planes is $\delta$;

- $\Sigma_{\delta}$ between adjacent reflection planes is "similar" to $S$.

Here the word "similar" is not well-defined in general. Since our goal is to approximate the reflection twin, we would expect the following asymptotic behavior for the sequence $\left(S_{\delta}\right)_{\delta \in \mathbb{N}}$ : There is a sequence of translations $\left(T_{\delta}\right)_{\delta \in \mathbb{N}}$ in the normal direction of $H$ such that $\left(S_{\delta} \cap T_{\delta}\left(H^{+}\right)\right)_{d \in \mathbb{N}}$ 
converges to $T_{\delta}\left(S \cap H^{+}\right)$. In our construction, this expectation is reflected either in the proposed Weierstrass representation (see Definition 3), or by the design of the Surface Evolver experiment (see Sections 3.5 and 4.1).

In the following, the word "polysynthetic" will be omitted unless otherwise stated.

\section{3. rPD TWIN SURFACES}

3.1. The rPD surfaces. The rPD-family is a 1-parameter family of TPMS with rhombohedral symmetries, generalizing Schwarz' D and P surfaces. A Weierstrass representation for the translational unit of an rPD surface is given in [Fogden, 1993] and [Fogden and Hyde, 1999]

$$
S_{t}: \omega \mapsto \operatorname{Re} \int^{\omega}\left(1-z^{2}, i\left(1+z^{2}\right), 2 z\right) R_{t}(z) d z
$$

where $\omega \in \mathbb{C}$,

$$
R_{t}(z)=\left[z\left(z^{3}-t^{3}\right)\left(z^{3}+t^{-3}\right)\right]^{-1 / 2},
$$

and $t>0$ is the parameter. The Schwarz' $\mathrm{D}$ and $\mathrm{P}$ surfaces are restored with $t=\sqrt{1 / 2}$ and $t=\sqrt{2}$, respectively. The rPD-family is self-conjugate; the conjugate surface of $S_{t}$ is $S_{1 / t}$.

The rPD surfaces are known to [Schwarz, 1972] and rediscovered by [Schoen, 1970] and [Karcher, 1989]. They belong to Meeks' 5-dimensional family [Meeks, 1990]. In the Weierstrass representation of a Meeks' surface, the Gauss map represents the translational unit as a two-sheeted cover of $\mathbb{S}^{2}$ with four antipodal pairs of simple branch points (eight in total). The branch points correspond to the flat points on the surface. For instance, after a stereographic projection onto the complex plane $\mathbb{C}$, the branch points of $S_{t}$ are the roots of $R_{t}(z)^{-2}$.

The Weierstrass data reflects the symmetries of the rPD surfaces. More specifically, the surface has vertical symmetry planes (parallel to the $z$-axis). The group generated by these reflections is the Euclidean triangle group with parameters $(3,3,3)$. [Weyhaupt, 2006] proved that an embedded TPMS of genus 3 with these symmetries must be an rPD surface or an $\mathrm{H}$ surface.

We recommend the following way to visualize rPD surfaces. Consider two equiangular triangles that intersect the $z$-axis perpendicularly at their centers, whose projections on the $x y$ plane differ by a rotation of $\pi / 3$. Our building block, which we call catenoid unit, is the "catenoid" spanned by the two triangles. The whole surface is obtained by the order- 2 rotations about the edges of the triangles. The $z$-axis is actually in the [111]-direction of the rhombohedral lattice.

Remark. If the projections of the two triangles coincide on the $x y$ plane, then the same construction yields an $\mathrm{H}$ surface. Catenoid units of $\mathrm{H}$ surfaces will appear on the twin boundaries of rPD twins.

The 1-parameter family rPD is obtained by "stretching" the two triangles along the $z$-axis. Let $h(t)$ denote the height of the catenoid unit (vertical distance between the triangles) assuming unit inradii for the triangles, and $A(t)$ be the area of the catenoid assuming unit total area for two triangles. Using the formulae given in Appendix A of [Fogden and Hyde, 1999] (where the parameter $r_{0}$ correspond to our $1 / t$ ), we plot $h$ and $A$ against $t$ in Figure 2. The calculations are done in Sage [The Sage Developers, 2016].

The height $h$ attains its maximum $h_{\max }=1.529295 \cdots$ at $t_{0}=0.494722 \cdots$ and converges to 0 in both limits $t \rightarrow \infty$ and $t \rightarrow 0$. With a distance bigger than $h_{\max }$, the triangles span no catenoid; in this case the only minimal surface is the relative interiors of the triangles. Any $h<h_{\max }$ corresponds to two different rPD surfaces.

The area $A$ attains its maximum $A_{\max }=1.163261 \cdots$ at $t=0.494893 \cdots$ and converges to 1 in the limit $t \rightarrow 0$, to $1 / 3$ in the limit $t \rightarrow \infty$. The area exceeds 1 as long as $t<t_{1}=0.877598 \cdots$ For $t$ between $t_{0}$ and $t_{1}$, the catenoid is only a local minimizer for the area functional; the relative interiors of the triangles has smaller area.

Other interesting points are: $t=\sqrt{1 / 2}$ for the $\mathrm{D}$ surface; $t=\sqrt{2}$ for the $\mathrm{P}$ surface; $t=1$ is a self-conjugate surface; and $t=1 / t_{0}$ is the rGPD surface according to [Fogden and Hyde, 1999]. 


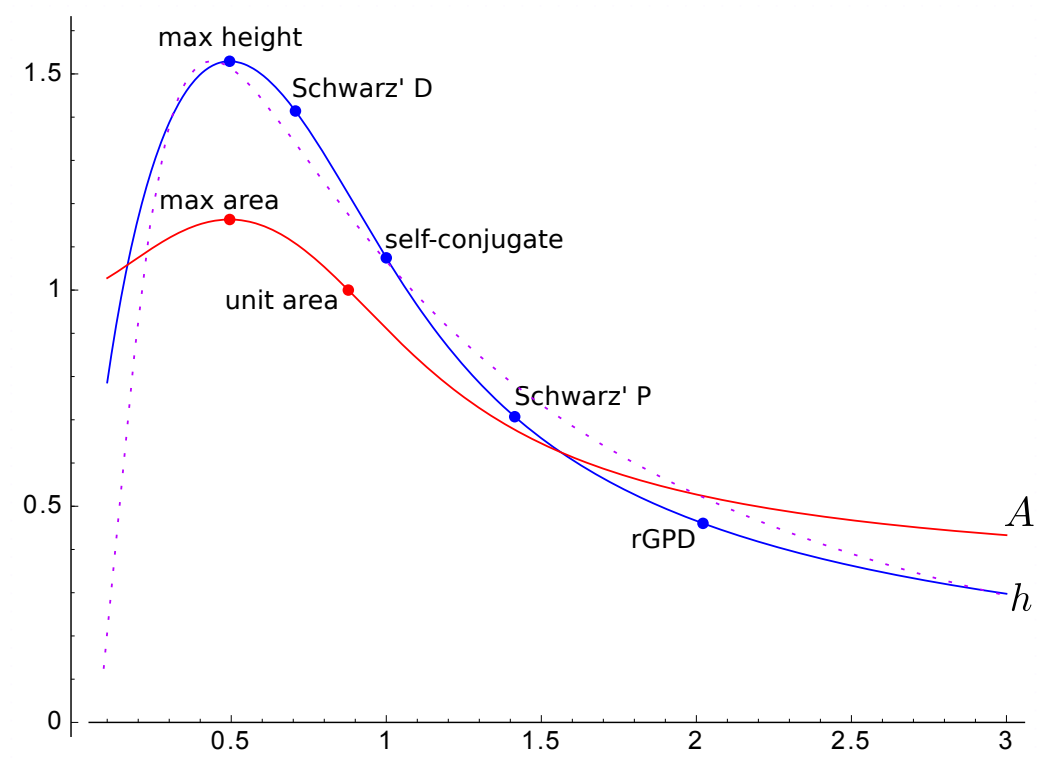

Figure 2. Plot of the height $h$ (solid blue) of a catenoid unit assuming unit inradii for the bounding triangles, and the area $A$ (solid red) assuming unit total area for the bounding triangles, against the parameter $t$ on the horizontal axis. The dotted line is the reparameterization of $h$ with the parameter $\tau$ in place of $t$ on the horizontal axis.

3.2. A Weierstrass representation of rPD twins. [Fujimori and Weber, 2009] developed an approach to construct surfaces with vertical symmetry planes. Instead of a cover of $\mathbb{S}^{2}$, the Gauss map descends to the quotient torus $\mathbb{C} /\langle 1, \tau i\rangle$, where $\tau \in \mathbb{R}_{+}$is an adjustable parameter. For example, the Weierstrass representation of an $\mathrm{rPD}$ surface is given by [Weyhaupt, 2006][Weyhaupt, 2008]

$$
S_{\tau}: \omega \mapsto \operatorname{Re} \int^{\omega}\left(\frac{1}{2}\left(G_{\tau}^{-1}-G_{\tau}\right), \frac{i}{2}\left(G_{\tau}^{-1}+G_{\tau}\right), 1\right) d z,
$$

where the Gauss map

$$
G_{\tau}(z)=\left(\rho \frac{\vartheta(z ; \tau)}{\vartheta(z-1 / 2-\tau / 2 ; \tau)}\right)^{2 / 3}
$$

is defined on the torus $\mathbb{C} /\langle 1, \tau i\rangle$. Here, $\rho$ is the Lopez-Ros factor, and

$$
\vartheta(z ; \tau)=\sum_{k=-\infty}^{\infty} e^{-\pi\left(k+\frac{1}{2}\right)^{2} \tau+2 \pi i\left(k+\frac{1}{2}\right)\left(z-\frac{1}{2}\right)}
$$

is one of the Jacobi $\vartheta$-functions. $\vartheta(z ; \tau)$ has simple zeros at the lattice points spanned by 1 and $\tau i$. At the bottom of Figure 3, we show the zeros and poles of $G_{\tau}$. They correspond to points of $S_{\tau}$ with vertical normal vectors; these are exactly the flat points of $S_{\tau}$, and lie on the intersections of the vertical symmetry planes.

Note that the parameter $\tau$ is different from $t$ but equivalent. For comparison, the height of the catenoid unit is plotted against $\tau$ by a dotted curve in Figure 2. The plot is generated by Sage [The Sage Developers, 2016], but the calculations are done using mpmath [Johansson et al., 2013]. The Schwarz' P surface is restored with $\tau=1.563401 \cdots$ [Weyhaupt, 2006]. The conjugate surface of $S_{\tau}$ is $S_{1 / \tau}$. We will take the liberty to switch between the two parameters whenever convenient.

Apart from the vertical reflection planes, horizontal reflection planes are assumed in [Fujimori and Weber, 2009] in order to take advantage of the symmetry. Up to a translation, we may assume that one horizontal symmetry plane correspond to the imaginary axis of $\mathbb{C}$. 


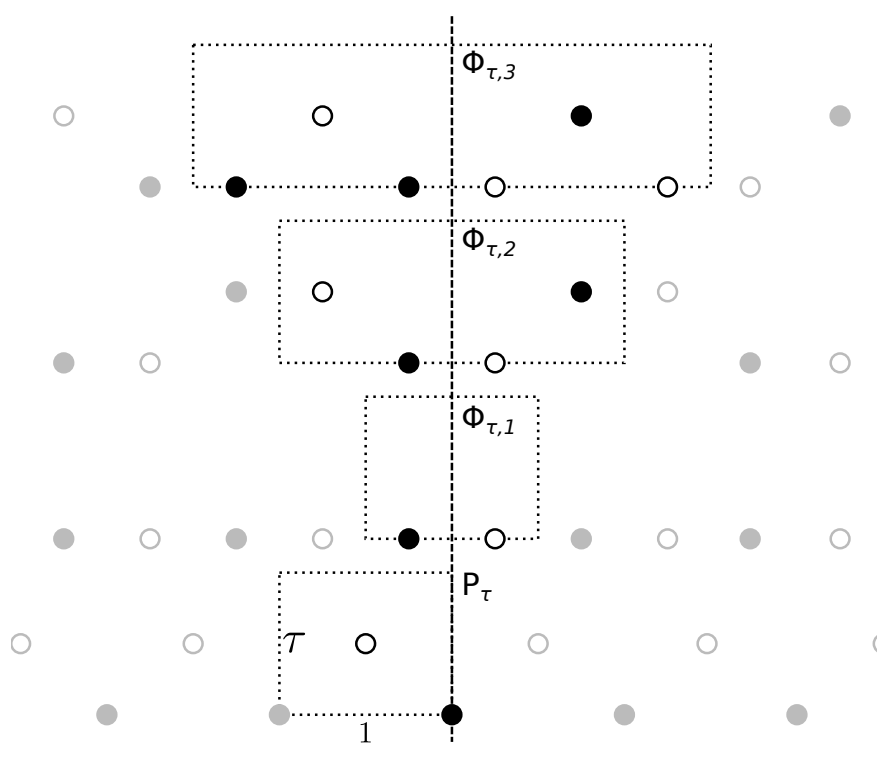

Figure 3. Zeros (filled circles) and poles (empty circles) of the Gauss maps $G_{\tau}$ and $G_{\tau, \delta}, \delta=1,2,3$. The dotted rectangles indicate the tori on which the Gauss maps are defined.

Following the argument in [Fujimori and Weber, 2009], we propose the following definition of rPD twins through Weierstrass representation.

Definition 3. The rPD twin surface $\Sigma_{\tau, \delta}$ is given by the Weierstrass representation

$$
\Sigma_{\tau, \delta}: \omega \mapsto \operatorname{Re} \int^{\omega}\left(\frac{1}{2}\left(G_{\tau, \delta}^{-1}-G_{\tau, \delta}\right), \frac{i}{2}\left(G_{\tau, \delta}^{-1}+G_{\tau, \delta}\right), 1\right) d z,
$$

where the Gauss map

$$
G_{\tau, \delta}(z)=\rho \prod_{k \text { odd }}\left(\frac{\vartheta\left(\left(z-p_{k}\right) / \delta ; \tau / \delta\right)}{\vartheta\left(\left(z+p_{k}\right) / \delta ; \tau / \delta\right)}\right)^{2 / 3} \prod_{k \text { even }}\left(\frac{\vartheta\left(\left(z-p_{k}-\tau / 2\right) / \delta ; \tau / \delta\right)}{\vartheta\left(\left(z+p_{k}-\tau / 2\right) / \delta ; \tau / \delta\right)}\right)^{-2 / 3}
$$

and $\left(p_{k}\right)_{1 \leq k \leq \delta}$ are real numbers such that $0<p_{1}<p_{2}<\cdots<p_{\delta}<\delta / 2$ and $p_{k}+p_{\delta+1-k}=\delta / 2$.

Note that we scale the $\vartheta$-function by $1 / \delta$ so that, within the stripe $0<\operatorname{Re}(z)<\delta / 2$, the zeros and poles of $G_{\tau, \delta}$ are similarly arranged as for $G_{\tau}$; see Figure 3. The Lopez-Ros factor $\rho$ is fixed to 1 so that $\left|G_{\tau, \delta}(z)\right|=1$ for all $z \in i \mathbb{R}$; see Appendix A of [Weyhaupt, 2006].

3.3. Example and non-examples of small $\delta$. The fact that $\Sigma_{\tau, \delta}$ satisfies the expected symmetries follows from [Fujimori and Weber, 2009]. But to prove their existence, we need to solve the period problem. In our case, the period problem for $\Sigma_{\tau, \delta}$ asks to find $p_{k}$ such that

$$
\operatorname{Re} \int^{p_{k}}\left(\frac{1}{2}\left(G_{\tau, \delta}^{-1}-G_{\tau, \delta}\right), \frac{i}{2}\left(G_{\tau, \delta}^{-1}+G_{\tau, \delta}\right)\right) d z
$$

are vertices of an equiangular triangle. In [Fujimori and Weber, 2009], the period problem is solved for small $\delta$ 's, as an answer is immediate by symmetry. When $\delta=1$ we have $p_{1}=1 / 4$; this is an $\mathrm{H}$ surface. When $\delta=2$, we have $p_{1}=1 / 4$ and $p_{2}=3 / 4$; this is Karcher's T-WP surface.

For $\delta=3$, the period problem is 1-dimensional. Examples of $\Sigma_{t, 3}$ are computed numerically. The Mathematica program for this purpose is kindly provided to us by Matthias Weber. The 
period problem asks to solve the following equations:

$$
\begin{aligned}
\operatorname{Re} \int_{0}^{3 / 2+\tau / 2} \frac{i}{2}\left(G_{\tau, 3}^{-1}+G_{\tau, 3}\right) d z & =0 \\
\cos \frac{\pi}{6} \operatorname{Re} \int_{\tau / 2}^{3 / 2} \frac{1}{2}\left(G_{\tau, 3}^{-1}-G_{\tau, 3}\right) d z+\sin \frac{\pi}{6} \operatorname{Re} \int_{\tau / 2}^{3 / 2} \frac{i}{2}\left(G_{\tau, 3}^{-1}+G_{\tau, 3}\right) d z & =0
\end{aligned}
$$

Under the symmetric assumptions $p_{2}=3 / 4$ and $p_{1}+p_{3}=3 / 2$, the lhs of the equations are actually the same.

When we change $\tau$ to a very large value, Mathematica can not find any root. In Figure 4 we plot the lhs of (1) against $p_{1} / 3$ for six values of $\tau$, which clearly shows absence of solution for $\tau \geq 3$. In these plots we allow $p_{1}>p_{2}=3 / 4$, in which case $p_{1}$ should be understood as $p_{3}$; the horizontal reflectional symmetry is then obvious. One observes that, as $\tau$ increases, $p_{1}$ and $p_{3}$ are pushed towards the center $p_{2}=3 / 4$, finally meet there and vanish. We calculate that $p_{1}=p_{2}=p_{3}=3 / 4$ when $\tau=\tau_{*}=2.916517 \cdots$.

Observation 2. The period problem for $\Sigma_{\tau, 3}$ has no solution for $\tau>\tau_{*}$.
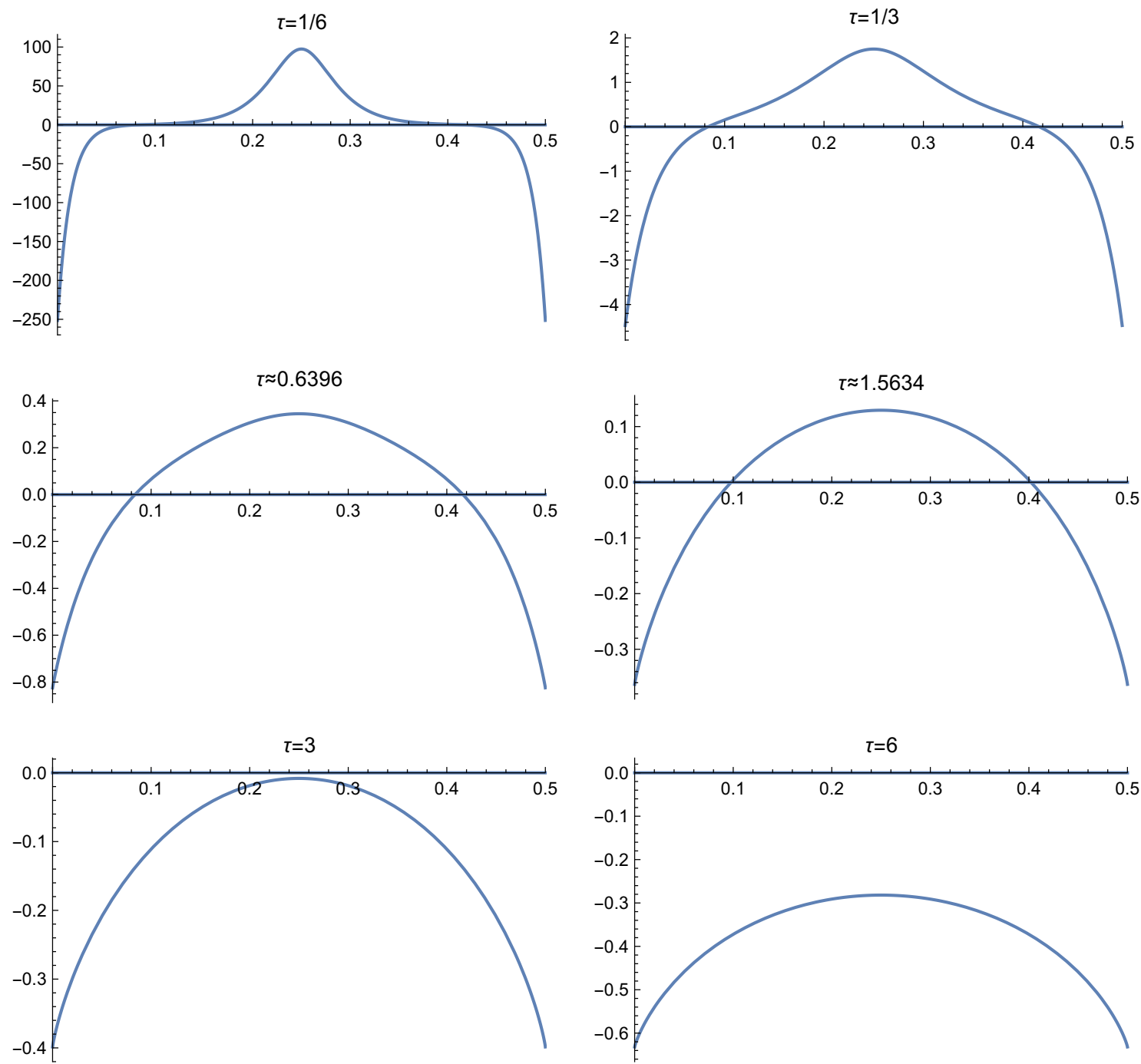

Figure 4. Plot of the lhs of (1) against $p_{1} / 3$ for six different $\tau$ 's. In the middle row, the left is the twin of $\mathrm{D}$ surface, the right is the twin of $\mathrm{P}$ surface. 
This observation is no surprise. The smaller the parameter $t$ is, the closer the catenoid unit is to a standard catenoid. A standard catenoid has a natural horizontal symmetry plane, hence it is easier to twin $S_{t}$ for small $t$. In the other limit $t \rightarrow \infty$, helicoids are forming. As the only properly embedded simply connected non-planar minimal surface in $\mathbb{R}^{3}$ [Meeks and Rosenberg, 2005], it is not possible to twin the standard helicoid. Therefore, twinning $S_{t}$ with large $t$ is expected to be difficult.

Another way to understand the situation is the following: On the one hand, when $t$ is large, $S_{t}$ is far from perpendicular to the horizontal planes. To meet the free boundary condition on the twin boundaries, a larger perturbation would be needed. Under the perturbation, the flat points at height $p_{1}$ and $p_{3}$ is moving vertically towards $p_{2}$. On the other hand, the flat points are already very close in the $z$ direction, and there is little space for perturbation. These two factors together reduce the possibility of twinning $\mathrm{rPD}$ surfaces with large $t$.

3.4. Examples of small $t$. In the other end, for any $\delta$, the period problem is solved by [Traizet, 2008 ] for sufficiently small $t$. We now introduce his work.

Traizet was interested in TPMS with periods $\left(T_{1}, 0\right),\left(T_{2}, 0\right)$ and $\left(T_{3}, \varepsilon\right)$ which looks like infinitely many horizontal planes $H_{0}, H_{1}, \cdots$ with $H_{0}$ at $z=0$ and $H_{N}$ at $z=\varepsilon$, and adjacent planes are connected by small catenoid necks. As $\varepsilon$ tends to 0 , such a TPMS converges to a countably sheeted plane, and the catenoid necks converges to singular points arranged in the lattice spanned by $T_{1}$ and $T_{2}$. We identify the plane to $\mathbb{C}$ so that we can talk about positions and periods by complex numbers. Let $m_{k}, 0 \leq k<N$, be the number of catenoid necks between $H_{k}$ and $H_{k+1}$ and $p_{k, i}$, $1 \leq i \leq m_{k}$, be the limit position of the $i$-th catenoid neck. The collection of $\left\{p_{k, i}\right\}$ together with the periods $T_{1}, T_{2}, T_{3}$ is called a configuration.

Given a configuration, [Traizet, 2008] defines the force on the $i$-th neck between $H_{k}$ and $H_{k+1}$ as follows:

$$
\begin{aligned}
F_{k, i}= & \sum_{j \neq i} \frac{2}{m_{k}^{2}} \zeta\left(p_{k, i}-p_{k, j}\right) \\
& -\sum_{k^{\prime}=k \pm 1} \sum_{j} \frac{1}{m_{k} m_{k^{\prime}}} \zeta\left(p_{k, i}-p_{k^{\prime}, j}\right) \\
& +\frac{1}{m_{k}}\left[\left(2 x_{k}-x_{k-1}-x_{k+1}\right) \eta_{1}+\left(2 y_{k}-y_{k-1}-y_{k+1}\right) \eta_{2}\right]
\end{aligned}
$$

where

$$
\zeta(z)=\frac{1}{z}+\sum_{0 \neq w \in\left\langle T_{1}, T_{2}\right\rangle}\left(\frac{1}{z-w}+\frac{1}{w}+\frac{z}{w^{2}}\right)
$$

is the Weierstrass $\zeta$ function; $\eta_{i}=2 \zeta\left(T_{i} / 2\right), i=1,2$; and the center of mass $\sum_{j} p_{k, j} / m_{k}=$ $x_{k} T_{1}+y_{k} T_{2}$. The configuration is said to be balanced if all the forces $F_{k, i}$ vanish, and nondegenerate if the differential of the map sending the positions $\left\{p_{k, i}\right\}$ to the forces $\left\{F_{k, i}\right\}$ has real co-rank 2. [Traizet, 2008] proved that, if the configuration is balanced and non-degenerate, then the TPMS described above exists and form a smooth family for sufficiently small $\epsilon$.

The rPD surfaces with small $t$ are examples of Traizet's surfaces; the configuration is given by

$$
\begin{gathered}
N=2, \quad m_{0}=m_{1}=1, \\
T_{1}=1, \quad T_{2}=a=\exp (i \pi / 3), \quad T_{3}=2(1+a) / 3, \\
p_{0,1}=0, \quad p_{1,1}=(1+a) / 3 .
\end{gathered}
$$

The balance and non-degeneracy has been proved in Section 4.3.3 of [Traizet, 2008]; see also Proposition 3 of the same paper.

The $\mathrm{H}$ surfaces are also examples; the only difference from the $\mathrm{rPD}$ surfaces is $T_{3}=0$. To verify Traizet's force balancing condition, consider the three integral of $\zeta^{\prime}(z)=-\wp(z)$ along the directed segments shown in Figure 5. The Weierstrass elliptic function $\wp(z)$ is even and invariant under the action of $\langle 1, a\rangle$. The integrals sum up to 0 because of the symmetries. That is,

$$
\left[\zeta\left(\frac{1+a}{3}\right)-\zeta\left(\frac{1}{2}\right)\right]+\left[\zeta\left(\frac{1+a}{3}\right)-\zeta\left(\frac{a}{2}\right)\right]+\left[\zeta\left(\frac{1+a}{3}\right)-\zeta\left(\frac{1+a}{2}\right)\right]=0 .
$$


Since $\zeta\left(\frac{1}{2}\right)+\zeta\left(\frac{a}{2}\right)=\zeta\left(\frac{1+a}{2}\right)$, we conclude that

$$
-2 \zeta\left(\frac{1+a}{3}\right)+\frac{4}{3} \zeta\left(\frac{1}{2}\right)+\frac{4}{3} \zeta\left(\frac{a}{2}\right)=0 .
$$

Hence the configuration is balanced. The non-degeneracy is verified numerically.

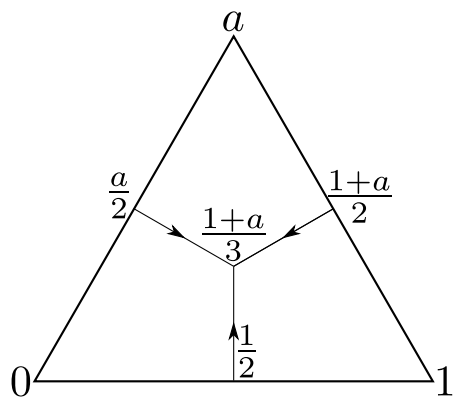

FIgURE 5. The integrals of $\zeta^{\prime}(z)=-\wp(z)$ along the three directed segments sum up to 0 because of the symmetry. This proves the force balancing condition for the $\mathrm{H}$ surfaces.

At this point, crystallographers should notice the analogy of rPD and $\mathrm{H}$ family with the cubic and hexagonal closed packings. Within a period, there are three possible limit positions for the necks, namely $j(1+a) / 3$ with $j=0,1,2$. We then obtain an infinite sequence consisting of letters 0,1 and 2 . The rPD surfaces has a periodic sequence $\cdots 012012012 \cdots$. The sequences for rPD twins should be periodic and palindromic, like $\cdots 010101010 \cdots$ for the $\mathrm{H}$ surface, $\cdots 012101210 \cdots$ for the T-WP surface, etc.

The formula (2) only involves adjacent layers. Up to symmetry, the only possible consecutive triples is 012 and 010, for which the force balancing condition has been verified in rPD and H surfaces. So the rPD twin configurations are all balanced and non-degenerate. Consequently, for any $\delta>0$, there is a real number $\epsilon_{\delta}>0$ such that the minimal twin surface $\Sigma_{\tau, \delta}$ exists for $0<\tau<\epsilon_{\delta}$. In fact, the argument works for any periodic sequence. For example, the sequence ..0101201012 $\cdots$ also satisfy the force balancing condition.

Traizet's proof uses implicit function theorem. We do not know if the following conjecture holds.

Conjecture 1. There is a positive number $\epsilon>0$ such that the $\mathrm{rPD}$ twin surface $\Sigma_{\tau, \delta}$ exists for all $0<\tau<\epsilon$ and $\delta>0$.

A proof of this conjecture would justify our strategy of using polysynthetic twins to approximate the single reflection twin.

3.5. Numerical examples with Surface Evolver. Traizet's result guarantees TPMS near the catenoid limit. It does not tell how small is sufficient. Sometimes $t$ doesn't need to be very small. In the case of rPD and H surfaces, the Traizet families extend all the way to the helicoid limit. For a concrete surface alleged in a Traizet family, we need numerical technique to confirm its existence.

Brakke's Surface Evolver [Brakke, 1992] is a software that simulates the physics of surfaces by minimizing energies. Surfaces are modeled by triangulations, and the energy is minimized by gradient descent method. Surface Evolver can handle various energies under various constraints.

In a usual application, the energy to minimize is the surface tension energy or the area functional. However, contrary to the intuition of many, TPMS do not result from area minimization. In the translational unit, a TPMS is actually a strict maximum of the area functional among its parallel surfaces [Große-Brauckmann, 2012]. A TPMS is indeed stable for the area functional if a volume constraint is imposed on the translational unit [Große-Brauckmann and Wohlgemuth, 1996]. But this is again false for any slightly larger piece [Ross, 1992]. Between two adjacent twin boundaries, $\Sigma_{t, \delta}$ correspond to a large piece of $S_{t}$, hence the area functional is not our option.

We will minimize instead the Willmore energy, or more precisely, the integral of the squared mean curvature; see [Hsu et al., 1992] for Surface Evolver experiments on this energy. Physically, 
the Willmore energy measures the deviation of the surface from the zero mean curvature, thus models the "bending" energy. The Willmore energy vanishes on minimal surfaces.

We construct the rPD twin surface $\Sigma_{t, \delta}$ in Surface Evolver in four steps:

(1) Prepare a catenoid unit.

Let $h=h(t)$ be the height function defined in Section 3.1. Consider four points

$$
A(1,0,0), B(-1 / 2, \sqrt{3} / 2,0), C(-1,0, h), D(1 / 2, \sqrt{3} / 2, h) \text {. }
$$

The catenoid unit of $S_{t}$ modulo reflectional symmetries is a minimal surface with fixed boundary conditions on the segments $A B$ and $C D$, and free boundary conditions on the vertical planes $y \pm \sqrt{3} x=\sqrt{3}$. Reflections in these planes yield the whole catenoid unit. The boundary triangles has unit inradius, compatible with the discussions in Section 3.1. The unit is obtained in Surface Evolver by minimizing the area functional. As a consequence of this practice, we can only obtain $\mathrm{rPD}$ surfaces with parameter $t>t_{0}=0.494722 \cdots$.

(2) Generate a slab.

We generate a slab of $S_{t}$ from the catenoid unit by order-2 rotations about the segment $A B$ or $C D$. In Surface Evolver, this is done by listing the matrices, say a and $\mathrm{b}$, of the two rotations in VIEW_TRANSFORM_GENERATORS. Assume an odd $\delta$, we set transform_expr to be a string $k(\mathrm{ba})$ if $\delta=4 k-1$ or $k(\mathrm{ba}) \mathrm{b}$ if $\delta=4 k+1$. Then the Surface Evolver will display $\delta+1$ catenoid unit. Finally, we use the detorus command to convert the displayed slab unit into a real surface.

(3) Slice the slab.

In this step, we use Brakke's script slicer.cmd. It is included in the Evolver distribution, and also available on the website of Surface Evolver. It removes the part of the surface on one side of a given plane. We slice the slab in the previous step by two horizontal planes with offset 0.5 at distance $\delta$ apart. The script also marks the vertices and edges newly created by the slice. This allows us to impose free boundary conditions by constraining the new vertices and edges on the slicing planes.

(4) Evolve the surface.

We turn off the surface tension energy (set facet tension 0), and turn on the Willmore energy, which is the integral of squared mean curvature. Then we leave Evolver to minimize the energy. Apart from the command $g$ that does one step of gradient descent, the command hessian_seek is particularly useful in this step to accelerate the calculation. If the Willmore energy decreases to practically 0 , we obtain a minimal surface with free boundary condition on the boundary of a triangular prism. Reflections in the faces of the prism give the whole $\Sigma_{t, \delta}$.

In Figure 6 , we show the result of each step with $t=\sqrt{2}$ (Schwarz' P surface) and $\delta=3$.

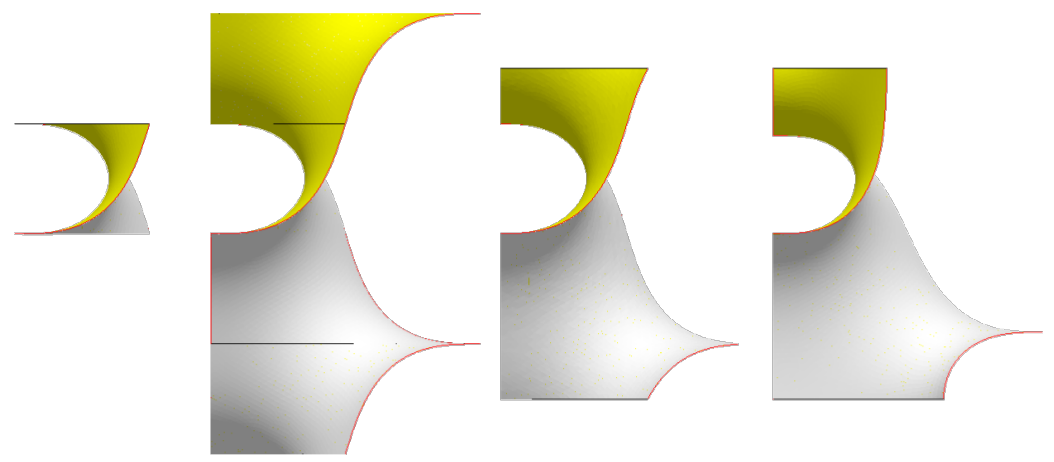

Figure 6. Result of each step with $t=\sqrt{2}$ and $\delta=3$.

The program can be easily modified to change the parameters $t$ and $\delta$. We perform calculations for parameters $t=t_{0}, \sqrt{1 / 2}(\mathrm{D}), 1, \sqrt{2}(\mathrm{P})$, and $\delta=1(\mathrm{H}), 3,5,11,21,99$. When $\delta$ is big, the last step will be very slow. We can increase the speed in the price of precision by reducing the number 


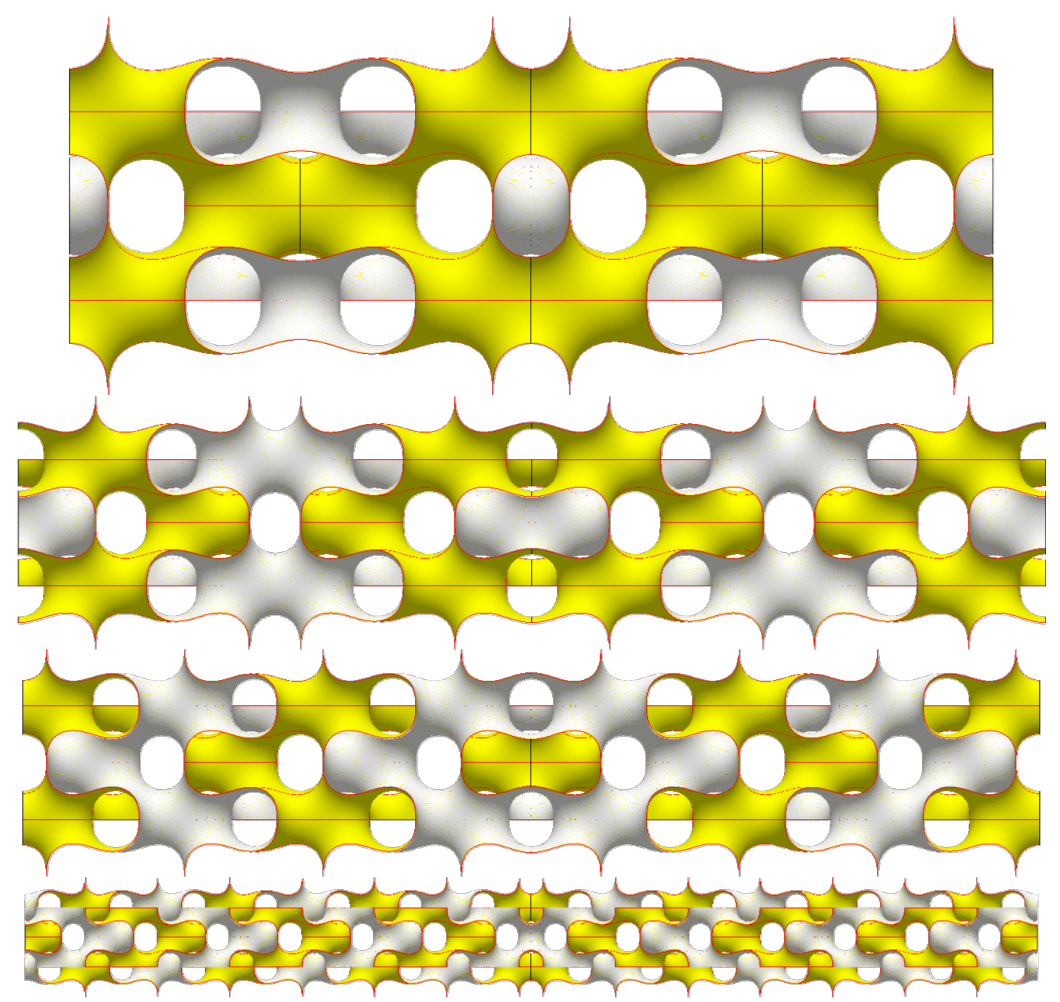

Figure 7 . D twin surfaces $(t=\sqrt{1 / 2})$. From top to bottom, $\delta=3,5,11,21$. The twin boundary is clearly seen in the middle.

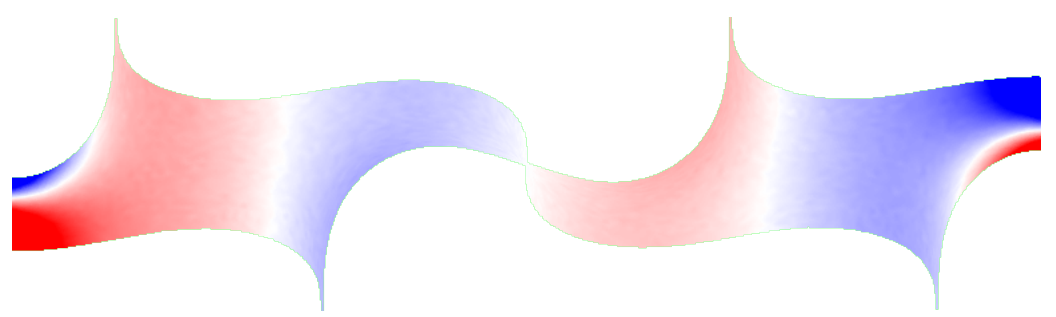

Figure 8. Deviation of the $\mathrm{D}$ twin with $\delta=5$ from the $\mathrm{D}$ surface. The picture shows the slab between two twin boundaries. Blue and red indicate perturbations in opposite directions; white indicates coincidence.

of faces in the catenoid unit. In Surface Evolver, the command $r$ refines the surface by subdividing each triangle into four. When generating the catenoid unit, we refine five times for $\delta=1,3,5$, four times for $\delta=11,21$ and three times for $\delta \geq 99$.

The calculation goes surprisingly smooth for small $t$ and small $\delta$. For $t=t_{0}$ and $t=\sqrt{1 / 2}(\mathrm{D})$, the Willmore energy decreases quickly to the order of $10^{-26}$ or lower, even when $\delta=99$. In view of the discussion in Section 3.3, it is reasonable to believe that the calculation would be even faster for smaller $t$. The results for $t=\sqrt{1 / 2}$ and $\delta=3,5,11,21$ are shown in Figure 7 . The difference from the D surface is not visible with human eyes. We use CloudCompare [Girardeau-Montaut et al., 2016] to calculate the deviation from the D surface; the result for $\delta=5$ is colored accordingly in Figure 8. We can see that the perturbation decays away from the twin boundaries, and the flat points are perturbed towards the middle. These pictures justify that the structures observed in [Han et al., 2011] are indeed minimal D twins.

Observation 3. The $\mathrm{rPD}$ twin surfaces $\Sigma_{t, \delta}$ exist for a large set of parameters. 
In fact, if we obtain a minimal surface, the calculation roughly solves the period problem. More specifically, the flat points are the vertices transformed from $A$ or $C$ when we generate the slab. They correspond to the poles and zeros of the Gauss map $G_{\tau, \delta}$. After normalization, the heights of these points give the sequence $\left(p_{k}\right)_{1 \leq k \leq \delta}$. For example, the $\Sigma_{\sqrt{1 / 2,3}}$ (D twin) generated by Surface Evolver has twin boundaries at $z= \pm 3 \sqrt{2} / 2$, and the surface intersects the lateral edges of the prism at $z \approx 0$ and $z \approx \pm 1.41240$. By a scaling and a translation, we may place the twin boundaries at $z=0$ and $z=3 / 2$, and obtain $p_{1} \approx 0.25064, p_{2} \approx 0.75$ and $p_{3} \approx 1.24936$. Compared to the solution given by the Mathematica program used for [Fujimori and Weber, 2009], these values are accurate until the fifth digit after the decimal point. However, the precision is not guaranteed as $t$ increases. The $\Sigma_{\sqrt{2}, 3}\left(\mathrm{P}\right.$ twin) shown in Figure 6 gives $p_{1} \approx 0.303350, p_{2} \approx 0.75$ and $p_{3} \approx 1.196651$, while Mathematica computes more precisely $p_{1}=0.293406 \cdots, p_{2}=0.75$ and $p_{3}=1.20659 \cdots$.

The Willmore energy also decreases to the order of $10^{-26}$ for $t=1$ with $\delta \leq 21$, and for $t=\sqrt{2}$ with $\delta \leq 3$. However, for $t=1$ and $\delta=99$, the Surface Evolver only manages to reduce the Willmore energy to the order of $10^{-8}$; then the decrement becomes extremely slow. For $t=\sqrt{2}$ (P surface) and $\delta=5$, the energy seems to stabilize at the order $10^{-4}$. These calculations are therefore inconclusive.

Observation 4. Surface Evolver does not converge to a minimal surface in a reasonable time if $t$ and $\delta$ is too large.

We then append more calculations for $t=\sqrt{1 / 2}$ to see if it eventually fails with sufficiently large $\delta$. It turns out that we are able to obtain a minimal surface with $\delta \leq 299$. Since the calculation is very time-consuming for large $\delta$, we did not perform further computation. But the success with large $\mathrm{D}$ twins ensures that the inconclusive results for $\mathrm{P}$ twins do not arise from numerical or system errors.

Remark. Despite the negative evidences, we refrain from conjecturing that $\mathrm{P}$ twins do not exist. Recently, [Mao et al., 2017] observed interconversions between P surface and D surface in their experiment. The structure looks like a P surface and a D surface glued along a (111) lattice plane. It suggests a possible mechanism of twinning the $\mathrm{P}$ surface through a $\mathrm{D}$ twin. However, it is too complicated to calculate the Weierstrass representation for large $\delta$, and Surface Evolver might be trapped in a local minimum of the Willmore energy, therefore won't reach such a configuration. So the approaches of this note wouldn't reveal such a twinning.

[Morabito and Traizet, 2012] and [Traizet, 2013] connected catenoid necks between countably many horizontal planes in a non-periodic way. In their works, every adjacent pair are connected by finitely many necks. But it is possible to extend their technique to connect necks between countably many tori in a non-periodic way ${ }^{3}$; see [Traizet, 2008]. This would lead to a rigorous construction for reflection $\mathrm{rPD}$ twins, as well as the $\mathrm{P}-\mathrm{D}$ interconversion and many other interesting structures, and would not involve any approximation as we hoped in Conjecture 1 . This will be the topic of a future project.

\section{G TWin SURFACES AND SOME SPECUlations}

4.1. Numerical examples with Surface Evolver. Twinnings of the G surface are also observed in experiment. Taking the flat points as lattice points, the observed twin boundaries are $\{211\}$ planes with offset 0 .

To generate $\mathrm{G}$ twin surfaces, we follow the same procedure for rPD twinning: Take a TPMS and impose free boundary condition on two parallel planes. If Surface Evolver is able to reduce the Willmore energy to practically 0 , then we obtain a minimal twin surface. A few modifications is however necessary.

(1) Prepare the G surface in an orthorhombic cell.

Unlike the rPD surfaces, the G surface contains no straight line, and has no symmetry plane. A datafile of initial triangulation is prepared by Fujita using the torus model of

\footnotetext{
${ }^{3}$ Confirmed by Traizet through personal communication.
} 
Surface Evolver. The orthorhombic unit cell is generated by three vectors in the [011], [111] and [211] directions. For our convenience, we made a small modification to place the [211] direction on the $z$-axis.

Since this unit cell is larger than the translational fundamental cell, it is not safe to minimize the area functional [Große-Brauckmann and Wohlgemuth, 1996]. We prepare the G surface by minimizing the Willmore energy directly.

(2) Generate a slab.

In the torus model, the command y 3 duplicates the displayed surface along the $z$-axis ([211]-direction). Repetition of the command for $k$ times would generate $2^{k}$ copies of the orthorhombic cell. We do not detorus the surface since we need the double periodicity.

(3) Slice the slab.

Brakke's script does not work well in the torus model. We write our own script to slice the slab by two horizontal planes ((211) planes) at offset 0 , and impose free boundary conditions on the slicing planes.

(4) Evolve the surface.

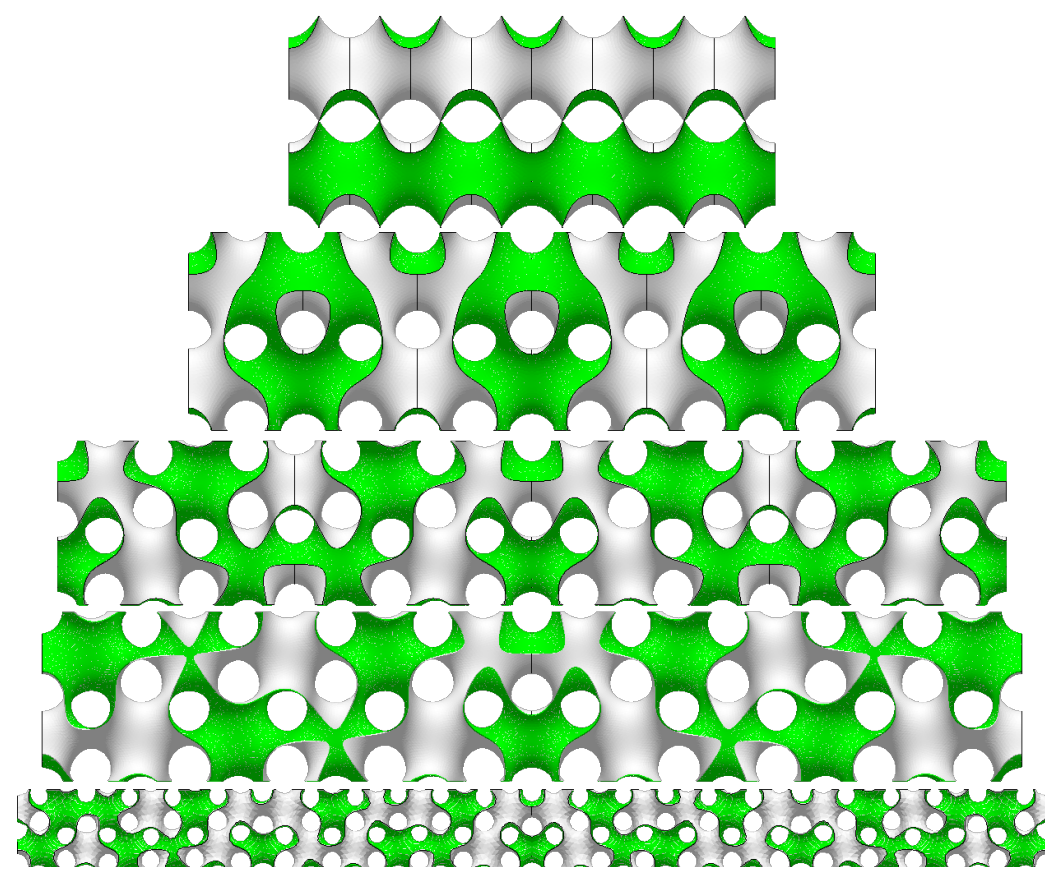

Figure 9. G twin surfaces. From top to bottom, $\delta=1,2,5,10,23$. The twin boundary is clearly seen in the middle.

The program can be easily modified to adjust the lattice distance $\delta$ between the slicing planes (twin boundaries). We perform calculation for $\delta=1,2,5,10,23$. In all these cases, we are able to obtain a minimal surface by reducing the Willmore energy to the order of $10^{-26}$. We did not try larger $\delta$. The results are shown in Figure 9. The G twin with $\delta=1$ turns out to be an orthorhombic deformation of the D surface (an oD surface); this will be explained later. In Figure 10, the result for $\delta=5$ is colored according to the deviation from the G surface.

Observation 5. The $\mathrm{G}$ twin surfaces exist for a large set of parameters.

4.2. Cubic polyhedral models. The Gyroid surface is probably the most beautiful TPMS, but has the reputation of being difficult to visualize. For Surface Evolver, a datafile written by Große-Brauckmann back in 1995 is still widely used to generate the G surface. It decomposes the cubic unit cell of the G surface into hexagons and rectangles, which is difficult to write from scratch. 


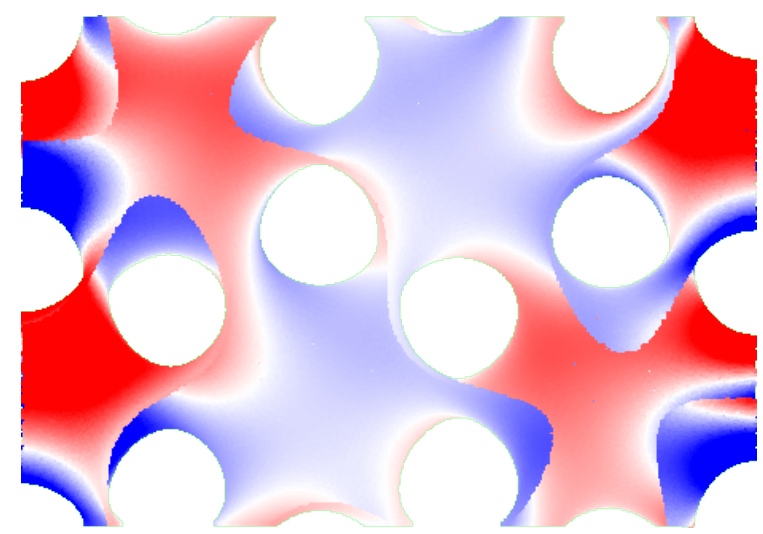

Figure 10. Deviation of the $\mathrm{G}$ twin with $\delta=5$ from the $\mathrm{G}$ surface. The picture shows the slab between two twin boundaries. Blue and red indicate perturbations in opposite directions; white indicates coincidence.

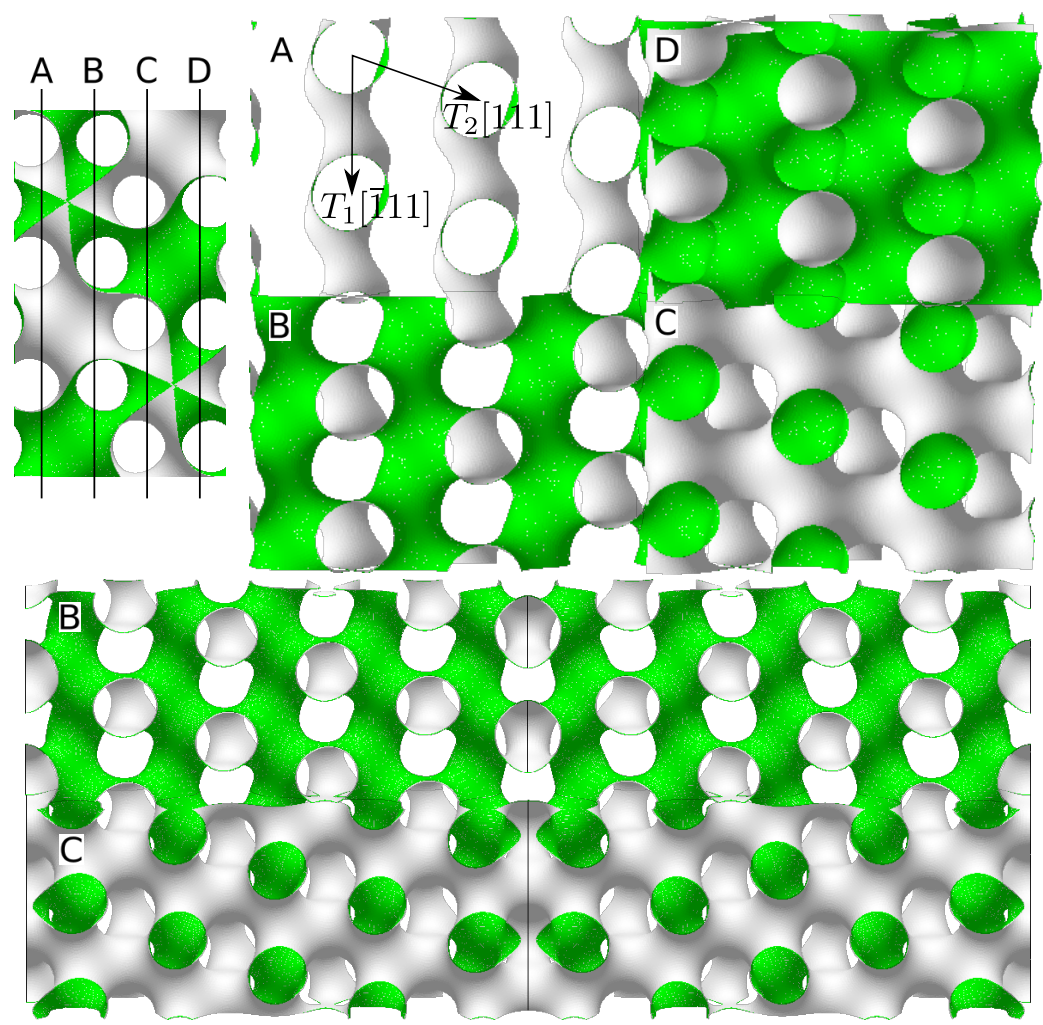

Figure 11. The G surface (upper right) and the $\mathrm{G}$ twin with $\delta=10$ (bottom) sliced by $(0 \overline{1} 1)$ planes at offset 0.5 . The position of the slicing planes are indicated in the upper left corner, where the G surface is seen in the [111] direction. In the upper right corner, intersection of the G surface with the slicing planes is highlighted by blue cycles.

The (211) G twins reveal an interesting structure of the G surface which, to the knowledge of the author, was not mentioned before. In the upper half of Figure 11 is a gyroid sliced by $(0 \overline{1} 1)$ planes at offset 0.5 . We observe necks arranged in a 2-D lattice spanned by vectors $T_{1}[\overline{1} 11]$ and $T_{2}$ [111], and the lattices in adjacent layers differ by $T_{1} / 2$ or $T_{2} / 2$, alternatively. In the lower half 
of the same figure, we show the slices of the $\mathrm{G}$ twin with $\delta=10$. We see clearly that the twin boundary is parallel to $T_{1}$ and perpendicular to the $(0 \overline{1} 1)$ planes.

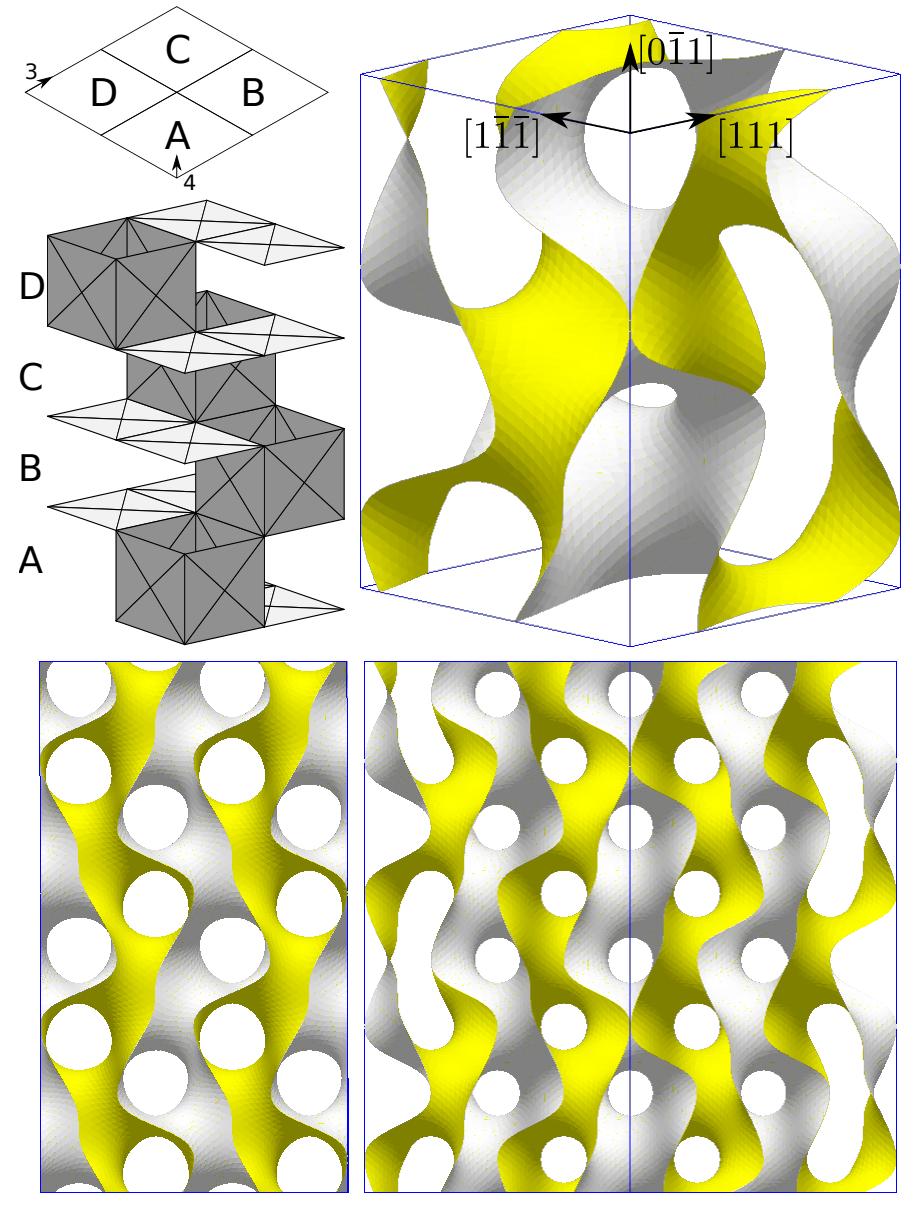

Figure 12. A new construction of the Gyroid in Surface Evolver. Upper left: the initial surface consists of four horizontal planes connected by tubes; see text. The rhombic tubes are labeled in accordance with slicing planes in Figure 11. The small arrows indicate the directions of 3- and 4-fold axis. Upper right: Evolved result in a monoclinic unit cell. Lower left: Evolved result seen along the 3-fold axis. Lower right: Evolved result seen along the 4-fold axis.

This observation leads to a new way to construct the G surface in Surface Evolver. The initial triangulation in torus model is shown in the upper left corner of Figure 12. The monoclinic unit cell is spanned by $( \pm \sqrt{2}, 1,0)$ and $(0,0,2 \sqrt{2})$; this cell has the same volume as the cubic cell. We prepare four horizontal planes at $z=j \sqrt{1 / 2}, j=0,1,2,3$. Each plane is a 2-torus decomposed into four rhombi. Vertical rhombic tubes connect adjacent planes as shown in the figure. After several refinements and evolvement, this simple configuration converges correctly to the G surface by minimizing the Willmore energy; see the evolved result in Figure 12.

Note that the initial triangulation is a discrete surfaces with quadrangle faces, containing all the vertices and edges from the monoclinic lattice. We call such surfaces cubic polyhedral. For cubic lattice, cubic polyhedral surfaces have been studied by [Goodman-Strauss and Sullivan, 2003] as a discrete model for Schwarz' P and CLP surfaces. Hence we just extended cubic polyhedral model to a monoclinic lattice for the $\mathrm{G}$ surface.

The rhombus spanned by $( \pm \sqrt{2}, 1,0)$ can be stretched along the diagonal (direction $(0,1,0)$, [100] in the gyroid lattice) into a rhombus spanned by $( \pm \sqrt{2}, c, 0)$. It becomes a square of side 
length 2 when $c=\sqrt{2}$. We then repeat the experiment with different values of $c$. The surface deforms continuously, and becomes the D surface when $c=\sqrt{2}$; see the right side of Figure 13 . The horizontal planes are (100) planes of the diamond lattice. Indeed, the diamond lattice has the symmetry of square lattice on (100) planes, and adjacent (100) planes differ by a half-period in alternating directions. We then obtain a new cubic polyhedral model for the $\mathrm{D}$ surface, and for every surface along this deformation.

Remark. In the experiments that produce Figure 13, the initial triangulation consists of two horizontal planes a triclinic unit cell spanned by $( \pm \sqrt{2}, c, 0)$ and $(0, c, \sqrt{2})$. Surface Evolver runs faster and better on this smaller unit cell.

The deformation above provides a continuous path from G to D. This seems strange because the $\mathrm{D}$ surface is a non-degenerate surface in the five-dimensional Meeks family, hence should stay in the family after small perturbations; but the $\mathrm{G}$ surface does not belong to Meeks family ${ }^{4}$. Indeed, a closer look reveals that the deformation above is not direct. As we increase $c$, the deformation seems to follow first the tG family (not in Meeks family), then the tD family (in Meeks family); see [Fogden and Hyde, 1999] for details about tD and tG families. We now present some evidences.

Note that the [100] direction is a 4 -fold axis of the $\mathrm{G}$ surface. If we reduce $c$, the $2 \mathrm{D}$ lattice is compressed into a very elongated rhombic lattice, and we obtain a 4-fold "saddle tower"; see the left side of Figure 13. This behavior is compatible with the tG family. The tunnels that we see through in Figure 13 correspond to helix curves in the surfaces. As we increase $c$, these tunnels shrink, since helix curves are becoming straighter. They eventually degenerate into straight lines as the deformation enters the tD family. Our experiment shows that the degeneracy occurs before we reach the $\mathrm{D}$ surface. We are not able to determine precisely where the degeneracy takes place, but it seems to be around the tGD surface as described in [Fogden and Hyde, 1999].

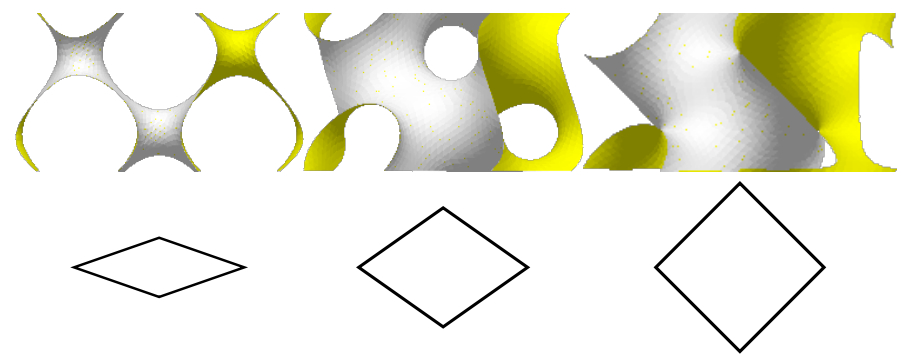

Figure 13. The diagonal of the rhombus (vertical in the figure) is a 4-fold axis of the $\mathrm{G}$ surface. By stretching the $\mathrm{G}$ surface (middle) in this direction, we obtain the D surface (right) and a 4-fold saddle tower (left). The surfaces shown in this figure are in the triclinic unit cell; the 4 -fold axis is towards the readers. To be compared with tG surfaces in Figure 11 of [Fogden and Hyde, 1999].

Remark. [Sadoc and Charvolin, 1989] observed that, by contracting edges, one can transform the skeleton of the $\mathrm{G}$ surface, into the skeleton of the D surface, and finally into the skeleton of $\mathrm{P}$. Between D and P, such transformation is explicitly given by the rPD family. Between G and D, our experiment suggest that such transformation is provided by the tG-tD path.

4.3. A monoclinic family of TPMS. Inspired by the experiments above, we notice the following family of Traizet configurations, which turns out to be balanced for any linearly independent $T_{1}$ and $T_{2}$ in $\mathbb{C}$, and is generically non-degenerate:

$$
\begin{aligned}
& N=2, \quad m_{0}=m_{1}=1, \\
& p_{0,1}=0, \quad p_{1,1}=T_{1} / 2, \\
& T_{3}=\left(T_{1}+T_{2}\right) / 2 .
\end{aligned}
$$

\footnotetext{
${ }^{4}$ The author thanks Mathias Weber and the anonymous referee for pointing this out.
} 
It follows from [Traizet, 2008] that each configuration (3) implies a family of TPMS near the catenoid limit, whose horizontal lattice planes admit the symmetry of the $2 \mathrm{D}$ lattice spanned by $T_{1}$ and $T_{2}$. Near Traizet's limit, we can also horizontally deform the rhombic lattice into other 2D lattices, as we did in the experiments above. In particular, there is a Traizet family $\left(T_{1,2}= \pm \sqrt{2}+i\right)$ whose horizontal planes has the symmetry of the (011) planes of the G surface, and another Traizet family $\left(T_{2}=i T_{1}\right)$ whose horizontal planes has the symmetry of the (100) planes of the D surface. We name these alleged deformations the $\mathrm{mG}$ family, since the surfaces have the symmetry of monoclinic lattices. In the neighborhood of a generic surface, this would be a three dimensional family: one dimension comes from the vertical deformations; the other two from the horizontal deformations.

One would naturally expect that every surface along the deformation path between the D and G surfaces belongs to a Traizet family. This seems not the case, at least not directly, for the D surface, as we now explain. We speculate the same phenomena for the G surface.

Note that the vertical direction ([100] in the diamond lattice) is the 4-fold axis of the D surface. Hence the Traizet family should preserve the tetragonal symmetry. We already know a tetragonal deformation family for the D surface, namely the tD family [Fogden and Hyde, 1999]. However, tD family admits a helicoid limit, but no catenoid limit. This suggests that the Traizet family and the tD family are not the same.

By extremely careful manipulations, we managed to produce both families in Surface Evolver; see Figure 14. The upper half of the figure is the tD family. We use $\mathrm{tD}^{\prime}$ to denote the Traizet family, shown in the lower half of the figure. The $\mathrm{tD}^{\prime}$ family is produced in Surface Evolver by explicitly connecting small necks between horizontal planes. Extreme caution has been taken to avoid crash.

The two families can be understood in the following way. In general, if two horizontal planes are close to each other, a catenoid neck between them can have two possible sizes. This phenomenon is famous for the catenoid, and also mentioned when we construct rPD surfaces. Our experiments shows that smaller necks correspond to the $\mathrm{tD}^{\prime}$ family, and larger necks correspond to the tD family.

As we increase the distance between (100)-planes, the tD and t $\mathrm{D}^{\prime}$ families seem to merge into one. We do not fully understand the behavior of surfaces near the junction. We propose the term extended tD family for the union of the two families.

We end this paper with some discussions on the twinning of $\mathrm{mG}$ surface. Near Traizet's catenoid limit, the $\mathrm{mG}$ surfaces can certainly be twinned about horizontal planes; the force balancing condition off the boundary is covered by (3), and the condition on the boundary is trivial. But in view of the observed $G$ twin, we are more interested in twinning $\mathrm{mG}$ surfaces about vertical planes parallel to $T_{1}$. We performed numerical calculations with $\delta=1,2,3$. However, if we insist that the twin boundaries pass through the catenoid necks, the only balanced configurations turn out to be (3) with $T_{1}=1, T_{2}=b i$ or $T_{2}=1+b i$, where $b=\sqrt{8 / 9}$. This is not exactly what we observed in the $\mathrm{G}$ twins; thus our understanding of $\mathrm{G}$ twins, and more generally $\mathrm{mG}$ twins, is still very limited. Note that the case $T_{2}=b i$ is an orthorhombic deformation of the D surface (oD) as we have observed, which should not be a surprise any more in view of previous discussions on the D surface.

For a concrete example of calculation, the configuration for $\delta=1$ is (up to scaling)

$$
\begin{aligned}
& N=4, \quad m_{k}=1 \quad(k=0 \ldots 3), \\
& p_{0,1}=0, \quad p_{1,1}=1 / 2, \\
& p_{2,1}=x+1 / 2+b i / 2, \quad p_{3,1}=x+b i / 2, \\
& T_{1}=1, \quad T_{2}=b i, \quad T_{3}=0 .
\end{aligned}
$$

The only solutions for $F_{0,1}=0$ are $x=0$ and $x=1 / 2$.

\section{REFERENCES}

[Brakke, 1992] Brakke, K. A. (1992). The surface evolver. Experiment. Math., 1(2):141-165. 


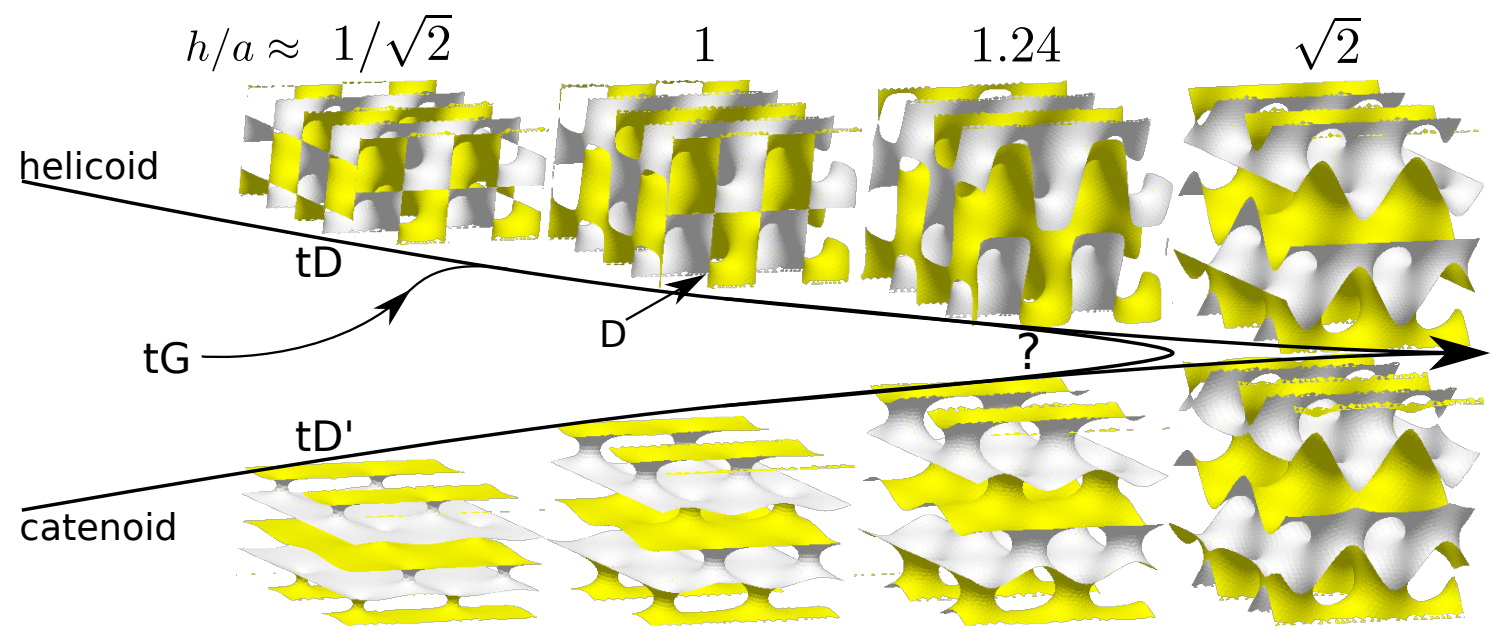

FiguRE 14. Surface Evolver simulations of the catenoid branch $\left(\mathrm{tD}^{\prime}\right)$ and the helicoid branch (tD) of the extended tD family, with different ratio of the vertical $(h)$ and horizontal $(a)$ lattice parameters. Surfaces in this figure has been obtained by minimizing the Willmore energy to practically 0. Extreme caution has been taken for the catenoid branch $\left(\mathrm{tD}^{\prime}\right)$ to avoid crash. The D surface is highlighted. Position where tG family joins tD is also sketched. Question mark at the junction indicates our ignorance.

[Fogden, 1993] Fogden, A. (1993). Parametrization of triply periodic minimal surfaces. III. General algorithm and specific examples for the irregular class. Acta Cryst. Sect. A, 49(3):409-421.

[Fogden and Hyde, 1999] Fogden, A. and Hyde, S. T. (1999). Continuous transformations of cubic minimal surfaces. The European Physical Journal B-Condensed Matter and Complex Systems, 7(1):91-104.

[Fujimori and Weber, 2009] Fujimori, S. and Weber, M. (2009). Triply periodic minimal surfaces bounded by vertical symmetry planes. Manuscripta Math., 129(1):29-53.

[Girardeau-Montaut et al., 2016] Girardeau-Montaut, D. et al. (2016). CloudCompare (Version 2.8.beta). Retrieved from http://www. cloudcompare.org/.

[Goodman-Strauss and Sullivan, 2003] Goodman-Strauss, C. and Sullivan, J. M. (2003). Cubic polyhedra. In Discrete geometry, volume 253 of Monogr. Textbooks Pure Appl. Math., pages 305-330. Dekker, New York.

[Große-Brauckmann, 2012] Große-Brauckmann, K. (2012). Triply periodic minimal and constant mean curvature surfaces. Interface Focus, 2:582-588.

[Große-Brauckmann and Wohlgemuth, 1996] Große-Brauckmann, K. and Wohlgemuth, M. (1996). The gyroid is embedded and has constant mean curvature companions. Calc. Var. Partial Differential Equations, 4(6):499-523. [Han et al., 2011] Han, L., Xiong, P., Bai, J., and Che, S. (2011). Spontaneous formation and characterization of silica mesoporous crystal spheres with reverse multiply twinned polyhedral hollows. Journal of the American Chemical Society, 133(16):6106-6109.

[Hsu et al., 1992] Hsu, L., Kusner, R., and Sullivan, J. (1992). Minimizing the squared mean curvature integral for surfaces in space forms. Experiment. Math., 1(3):191-207.

[Hyde et al., 1996] Hyde, S., Blum, Z., Landh, T., Lidin, S., Ninham, B., Andersson, S., and Larsson, K. (1996). The Language of Shape. Elsevier.

[Johansson et al., 2013] Johansson, F. et al. (2013). mpmath: a Python library for arbitrary-precision floating-point arithmetic (version 0.18). http://mpmath.org/.

[Karcher, 1989] Karcher, H. (1989). The triply periodic minimal surfaces of Alan Schoen and their constant mean curvature companions. Manuscripta Math., 64(3):291-357.

[Lin et al., 2017] Lin, Z., Liu, S., Mao, W., Tian, H., Wang, N., Zhang, N., Tian, F., Han, L., Feng, X., and Mai, Y. (2017). Tunable self-assembly of diblock copolymers into colloidal particles with triply periodic minimal surfaces. Angewandte Chemie International Edition, 56(25):7135-7140.

[Mao et al., 2017] Mao, W., Cao, X., Sheng, Q., Han, L., and Che, S. (2017). Silica scaffold with shifted plumber's nightmare networks and their interconversion into diamond networks. Angewandte Chemie International Edition, 56(36):10670-10675.

[Meeks, 1990] Meeks, III, W. H. (1990). The theory of triply periodic minimal surfaces. Indiana Univ. Math. J., 39(3):877-936.

[Meeks and Rosenberg, 2005] Meeks, III, W. H. and Rosenberg, H. (2005). The uniqueness of the helicoid. Ann. of Math. (2), 161(2):727-758. 
[Morabito and Traizet, 2012] Morabito, F. and Traizet, M. (2012). Non-periodic Riemann examples with handles. Adv. Math., 229(1):26-53.

[Ross, 1992] Ross, M. (1992). Schwarz' P and D surfaces are stable. Differential Geom. Appl., 2(2):179-195.

[Sadoc and Charvolin, 1989] Sadoc, J.-F. and Charvolin, J. (1989). Infinite periodic minimal surfaces and their crystallography in the hyperbolic plane. Acta Cryst. Sect. A, 45(1):10-20.

[Schoen, 1970] Schoen, A. H. (1970). Infinite periodic minimal surfaces without self-intersections.

[Schwarz, 1972] Schwarz, H. A. (1972). Gesammelte mathematische Abhandlungen. Band I, II. Chelsea Publishing Co., Bronx, N.Y. Nachdruck in einem Band der Auflage von 1890.

[The Sage Developers, 2016] The Sage Developers (2016). SageMath, the Sage Mathematics Software System (Version 7.3). http://www. sagemath.org.

[Traizet, 2008] Traizet, M. (2008). On the genus of triply periodic minimal surfaces. J. Differential Geom., 79(2):243275 .

[Traizet, 2013] Traizet, M. (2013). Opening infinitely many nodes. J. Reine Angew. Math., 684:165-186.

[Vignolini et al., 2012] Vignolini, S., Yufa, N. A., Cunha, P. S., Guldin, S., Rushkin, I., Stefik, M., Hur, K., Wiesner, U., Baumberg, J. J., and Steiner, U. (2012). A 3d optical metamaterial made by self-assembly. Advanced Materials, 24(10):OP23-OP27.

[Weyhaupt, 2006] Weyhaupt, A. G. (2006). New families of embedded triply periodic minimal surfaces of genus three in euclidean space. ProQuest LLC, Ann Arbor, MI. Thesis (Ph.D.)-Indiana University.

[Weyhaupt, 2008] Weyhaupt, A. G. (2008). Deformations of the gyroid and Lidinoid minimal surfaces. Pacific J. Math., 235(1):137-171

Mathematical Sciences Research Institute, 17 Gauss Way, Berkeley, 94720 California, USA

E-mail address: hao.chen.math@gmail.com 